\title{
Balanced per capita contributions and level structure of cooperation*
}

\author{
María Gómez-Rúa \\ Departamento de Estatística e Investigación Operativa \\ Universidade de Vigo \\ Juan Vidal-Puga ${ }^{\dagger}$ \\ Research Group in Economic Analysis and \\ Departamento de Estatística e Investigación Operativa \\ Universidade de Vigo
}

October 20, 2009

\begin{abstract}
We define a new value for games with a level structure and introduce a new property in these kind of games, called balanced per capita contributions, related with other properties in the literature. Further, we provide an axiomatic characterization of this value using this new property.
\end{abstract} tions.

Keywords: level structure, value, balanced per capita contribu-

\section{Introduction}

In many real situations the agents cooperate in order to get a benefit. These situations can be modelled as a transferable utility (TU, for short) game in

*Financial support from the Spanish Ministerio de Ciencia y Tecnología and FEDER through grant SEJ2005-07637-C02-01/ECON and the Xunta de Galicia through grants PGIDIT06PXIC300184PN and PGIDIT06PXIB362390PR is gratefully acknowledged.

${ }^{\dagger}$ Corresponding author. E-mail: vidalpuga@uvigo.es 
which the players divide themselves into groups for the purpose of bargaining. In addition, these situations are modelled as TU games with coalition structures. In many situations, however, a coalition structure does not provide a complete description of the cooperation structure. For instance, consider the members of the European Union Parliament. In this situation, even though they all have the same rights, they do not act independently. The natural cooperation structure will be formed by the parties. However, on a higher level, parties may associate according to their ideology in larger groups, such as the European People's Party (EPP), the European Democrats (ED), the Party of European Socialist (PES), etc. In an even higher level, the EPP and the ED form a larger group, the EPP-ED, and other groups so forth. This example appears in Winter (1989), Calvo et al. (1996) and Vidal-Puga (2005). In situations like this, a more detailed mapping of the cooperation structure is needed. This cooperation description of the players is called a level structure. There are several values in the literature, that take into account the level structure. For the particular case of the one single level, Aumann and Drèze (1974) first proposed a value for these kind of games and Owen (1977) defined a new value, the Owen value. Both of these values extend the Shapley value (Shapley, (1953b)). In addition, other extensions are provided by Hamiache (2006) and Kamijo (2007). On the other hand, Levy and McLean (1989) proposed a value that is an extension of the weighted Shapley value (Kalai and Samet (1984)).

Winter (1989) defined a value, the level structure value, first suggested by Owen in 1977 which is an extension of the Owen value for several levels. Calvo et al. (1996) then provided a characterization of the level structure value using the principle of balanced contributions. This property states that, for any two coalitions that belong to the same coalition at higher levels, the amount that the players in each coalition would gain or lose by the other coalition's withdrawal from the game should be equal.

Nevertheless, when the coalitions represent groups of different sizes, this symmetry among coalitions may not always be a reasonable requirement for a value. See, for instance, Levy and McLean (1989) or Kalai and Samet (1987) for further reading. The same argument was considered in Vidal-Puga (2006) who defined a value $\zeta$ for games with a unique level of cooperation taking into account the asymmetry between the coalitions due to their different sizes.

In this paper we extend the value $\zeta$ for games with a level structure. Moreover, we introduce a new property for these kind of games, balanced per capita contributions, that is related to another property proposed by 
Myerson (1980), called $\lambda$-balanced contributions, and also studied by Hart and Mas-Colell (1989), Sánchez (1997) and Calvo and Santos (2000).

The property of balanced per capita contributions states that for any two coalitions that belong to the same coalition at higher levels, the average amount that players in each coalition would gain or lose by the other coalition's withdrawal from the game should be equal. The average is taken over the number of single agents in each coalition.

We also provide a characterization of the new value using this property.

A similar axiom was introduced in Herings et al. (2008) in the context of games with a structure of graphs with no cycles. The property they introduced is component fairness and states that deleting a link between two players yields for both resulting components the same average change in payoff, where the average is taken over the players in the component. This property is related to the property of fairness (Myerson, 1977) that states that deleting a link between two players yields the same change in payoff for both of them.

The paper is organized as follows. In Section 2 we introduce the model. In Section 3 we introduce a new value for these kind of games. In Section 4 we define the property of balanced per capita contributions. Moreover, we prove that the new value satisfies this property. In Section 5 we provide a characterization of the value.

\section{The model}

A game with transferable utility, $T U$ game, is a pair $(N, v)$, where $N$ is finite and $v: 2^{N} \rightarrow \mathbb{R}$ satisfies $v(\emptyset)=0$. We denote by $T U(N)$ the set of $T U$ games with $N$ as player set. If $S \subset N$, we denote by $(S, v)$ the restriction of $(N, v)$ to the player set $S$. Given $S \subset N$, we denote $f_{S}(N, v)=\sum_{i \in S} f_{i}(N, v)$.

Given $N$ finite, we call coalition structure on $N$ a partition of the player set $N$, i.e. $\mathcal{C}=\left\{C_{1}, C_{2}, \ldots, C_{m}\right\} \subset 2^{N}$ is a coalition structure if it satisfies $\bigcup_{C_{q} \in \mathcal{C}} C_{q}=N$ and $C_{q} \cap C_{r}=\emptyset$ when $q \neq r$. We also assume $C_{q} \neq \emptyset$ for all $q$. Given $S \subset N$, we denote by $\mathcal{C}_{S}$ the coalition structure restricted to $S$, i.e. $\mathcal{C}_{S}=\left\{C_{q} \cap S: C_{q} \in \mathcal{C}, C_{q} \cap S \neq \emptyset\right\}$.

A level structure for $N$ is a sequence $\mathfrak{C}=\left(\mathcal{C}^{0}, \mathcal{C}^{1}, \ldots, \mathcal{C}^{h}\right), h \geq 1$ with $\mathcal{C}^{l}$ $(0 \leq l \leq h)$ coalition structure on $N$ such that:

1. $\mathcal{C}^{0}=\{\{1\},\{2\}, \ldots,\{n\}\}$. 
2. $\mathcal{C}^{h}=\{N\}$.

3. If $C_{q}^{l} \in \mathcal{C}^{l}$ with $0<l \leq h$ then $C_{q}^{l}=\bigcup_{S \in \mathcal{Q}} S$ for some $\mathcal{Q} \subset \mathcal{C}^{l-1}$.

Denote $\mathcal{C}^{l}=\left\{C_{1}^{l}, \ldots, C_{m(l)}^{l}\right\}$ and let $N^{l}=\{1, \ldots, m(l)\}$. We call $\mathcal{C}^{l}$ the $l$-th level of $\mathfrak{C}$. We say that $\mathfrak{C}$ is a level structure of degree $h$. Hence, the level structure $\mathfrak{C}$ has $h+1$ levels.

If $h=1$, we say that $\mathfrak{C}$ is a trivial level structure.

Given $\mathcal{C}^{l} \in \mathfrak{C}$ we define $\mathfrak{C} / \mathcal{C}^{l}$ as the level structure induced from $\mathfrak{C}$ by considering the coalitions in $\mathcal{C}^{l}$ as players. In this case, we denote the players in $\mathcal{C}^{l}$ by their indices. Formally, $\mathfrak{C} / \mathcal{C}^{l}=\left(\mathcal{D}^{l}, \mathcal{D}^{l+1}, \ldots, \mathcal{D}^{l+1}\right)$ where $\mathcal{D}^{l}=\{\{1\},\{2\}, \ldots,\{m(l)\}\}$ and, in general, for each $k(l \leq k \leq h)$, $\mathcal{D}^{k}=\left\{D_{1}^{k}, D_{2}^{k}, \ldots, D_{m(k)}^{k}\right\}$ with $D_{q}^{k}=\left\{r: C_{r}^{l} \subset C_{q}^{k}\right\}$ for all $q(1 \leq q \leq m(k))$.

Let $L T U$ be the set of all $T U$ games with a level structure. When the level structure is clear, we may write $(N, v)$ or $v$ instead of $(N, v, \mathfrak{C})$.

The quotient game $\left(N^{l}, v / \mathcal{C}^{l}, \mathfrak{C} / \mathcal{C}^{l}\right)$ is the game in $L T U$ defined on the coalition structure $\mathcal{C}^{l}$ with characteristic function

$$
\left(v / \mathcal{C}^{l}\right)(Q)=v\left(\bigcup_{q \in Q} C_{q}^{l}\right)
$$

for all $Q \subset N^{l}$.

A value in $L T U$ is a function $f$ that assigns to each $(N, v, \mathfrak{C}) \in L T U$ a vector $f(N, v, \mathfrak{C}) \in \mathbb{R}^{N}$. As usual, $f_{i}(N, v, \mathfrak{C})$ represents the payoff received by player $i \in N$.

A value $f$ is efficient if $\sum_{i \in N} f_{i}(N, v)=v(N)$ for each $v \in T U(N)$.

One of the most important values in $T U$ games is the Shapley value (Shapley (1953b)). We denote the Shapley value of the $T U$ game $v$ as $S h(v)$.

A nonsymmetric generalization of the Shapley value is the weighted Shapley value (Shapley (1953a), Kalai and Samet $(1987,1988))$. Given a vector of weights $\omega \in \mathbb{R}_{++}^{N}$, we denote the weighted Shapley value as $S h^{\omega}(v)$.

\section{A new value}

Winter (1989) defined the level structure value (LSV), that is an extension of the Owen value for this kind of games. 
One of the properties that is satisfied by the $L S V$ is coalitional symmetry (Winter, 1989, page 229). This property ensures that if two coalitions are symmetric in the quotient game and also they belong to the same coalition in the next level; their members should receive the same aggregate amount. There are several authors in the literature that claim that when the coalitions represent groups of different size, this symmetry may not be a reasonable requirement. See, for instance, Levy and McLean (1989) or Kalai and Samet (1987). In particular, the latter claimed that in this case, it seems reasonable to assign a size-depending weight to each coalition.

The value $\zeta$ presented by Vidal-Puga (2006) for $T U$ games with coalition structure takes this idea into account.

Now we extend the value $\zeta$ for games with a level structure. The intuitive idea of this value is as follows: In a first stage, we distribute $v(N)$ among the coalitions of the $(h-1)$-th level through the weighted Shapley value with weights given by the size of the coalitions. Then, for any $C_{q}^{h-1} \in \mathcal{C}^{h-1}$, we distribute the payment received by $C_{q}^{h-1}$ in the first stage among all the coalitions in $\mathcal{C}^{h-2}$ that belong to $C_{q}^{h-1}$. We also distribute this payment through the weighted Shapley value. In the last stage, we distribute the payment received by the coalitions in $\mathcal{C}^{1}$ among the agents.

The formal mechanism to compute the value is as follows: Let $(N, v, \mathfrak{C})$ with $\mathfrak{C}=\left(\mathcal{C}^{0}, \mathcal{C}^{1}, \ldots, \mathcal{C}^{h}\right)$. Take $v^{h}=v$.

- Stage 1: Take the $(h-1)$-th level $\mathcal{C}^{h-1}=\left\{C_{1}^{h-1}, \ldots ., C_{m(h-1)}^{h-1}\right\}$ and $N^{h-1}=\{1, \ldots, m(h-1)\}$. Let $C_{q}^{h-1} \in \mathcal{C}^{h-1}$. For each $T \subset C_{q}^{h-1}$, consider a weight system $\delta(T) \in \mathbb{R}_{++}^{N^{h-1}}$ given by $\delta_{q}(T)=|T|$ and $\delta_{r}(T)=\left|C_{r}^{h-1}\right|$ otherwise. The $T U$ game $\left(C_{q}^{h-1}, v^{h-1}\right)$ is defined as

$$
v^{h-1}(T)=S h_{q}^{\delta(T)}\left(N^{h-1}, v^{h} / \mathcal{C}_{\left(N \backslash C_{q}^{h-1}\right) \cup T}^{h-1}\right) .
$$

In particular, $v^{h-1}\left(C_{q}^{h-1}\right)$ is the amount assigned to coalition $C_{q}^{h-1}$.

- Stage 2: Take the $(h-2)$-th level $\mathcal{C}^{h-2}=\left\{C_{1}^{h-2}, \ldots, C_{m(h-2)}^{h-2}\right\}$ with $C_{r}^{h-2} \in \mathcal{C}^{h-2}$ and $N^{h-2}=\{1, \ldots, m(h-2)\}$. Let $C_{q}^{h-1} \in \mathcal{C}^{h-1}$ and $C_{r}^{h-2} \subset C_{q}^{h-1}$. Let $N_{q}^{h-2}=\left\{s \in N^{h-2}: C_{s}^{h-2} \subset C_{q}^{h-1}\right\}$ be the set of indices that define the coalitions in $C_{q}^{h-1}$. Notice that $r \in N_{q}^{h-2}$. For each $T \subset C_{r}^{h-2}$, consider a weight system $\delta(T) \in \mathbb{R}_{++}^{N_{q}^{h-2}}$ given by 
$\delta_{r}(T)=|T|$ and $\delta_{p}(T)=\left|C_{p}^{h-2}\right|$ otherwise. The $T U$ game $\left(C_{r}^{h-2}, v^{h-2}\right)$ is defined as

$$
v^{h-2}(T)=S h_{r}^{\delta(T)}\left(N_{q}^{h-2}, v^{h-1} / \mathcal{C}_{\left(C_{q}^{h-1} \backslash C_{r}^{h-2}\right) \cup T}^{h-2}\right) .
$$

In particular, $v^{h-2}\left(C_{r}^{h-2}\right)$ is the amount assigned to coalition $C_{r}^{h-2}$.

- Stages 3 through $h-1$ : We define the $T U$ game $\left(C_{s}^{l}, v^{l}\right)$ for each $\mathcal{C}^{l}$ and each $C_{s}^{l} \in \mathcal{C}^{l}$ as before $(l=h-3, \ldots, 1)$.

In particular, $v^{l}\left(C_{s}^{l}\right)$ is the amount assigned to coalition $C_{s}^{l}$.

- Stage h: Take $\mathcal{C}^{0}=\left\{C_{1}^{0}, \ldots, C_{n}^{0}\right\}$ with $C_{i}^{0}=\{i\}$, and $N^{0}=N$. Let $C_{q}^{1} \in \mathcal{C}^{1}, i \in C_{q}^{1}$, and $N_{q}^{0}=\left\{j \in N^{0}: j \in C_{q}^{1}\right\}$. The amount assigned to player $i$ is given by

$$
\phi_{i}(N, v, \mathfrak{C})=S h_{i}\left(N_{q}^{0}, v^{1} / \mathcal{C}_{C_{q}^{1}}^{0}\right) .
$$

It is well-known that the (weighted) Shapley value is additive ${ }^{1}$. Hence, it follows from this definition that $\phi$ is additive, as well.

If $h=1$, both $L S V$ and $\phi$ coincide with $S h$; whereas, for $h=2$, the $L S V$ coincides with the Owen value and $\phi$ coincides with $\zeta$.

Example 1 Consider the game $\left(N, u_{S}, \mathfrak{C}\right)$, where $N=\{1,2,3,4\}, \mathfrak{C}=\left\{\mathcal{C}^{0}, \mathcal{C}^{1}, \mathcal{C}^{2}, \mathcal{C}^{3}\right\}$ such that $\mathcal{C}^{1}=\{\{1\},\{2,3\},\{4\}\}, \mathcal{C}^{2}=\{\{1,2,3\},\{4\}\}$ and carrier $S=$ $\{1,2,4\}$. The denote $C_{1}^{1}=\{1\}, C_{2}^{1}=\{2,3\}$ and so on; so that $N^{1}=\{1,2,3\}$ and $N^{2}=\{1,2\}$.

We will detail the stages one by one.

Stage 1: In this case, we have two games $v^{2}$, one for each coalition in $\mathcal{C}^{2}$. The game $\left(\{4\}, v^{2}\right)$ is defined as $v^{2}(\{4\})=S h_{2}^{(3,1)}\left(\{1,2\}, v / \mathcal{C}_{N}^{2}\right)=\frac{1}{4}$, whereas $^{2}$ the game $\left(\{1,2,3\}, v^{2}\right)$ is defined as $v^{2}(\{1\})=S h_{1}^{(1,1)}\left(\{1,2\}, v / \mathcal{C}_{\{1,4\}}^{2}\right)=$ 0 ; and analogously $v^{2}(\{2\})=v^{2}(\{3\})=0, v^{2}(\{1,2\})=S h_{1}^{(2,1)}\left(\{1,2\}, v / \mathcal{C}_{\{1,2,4\}}^{2}\right)=$ $\frac{2}{3}, v^{2}(\{1,3\})=v^{2}(\{2,3\})=0, v^{2}(\{1,2,3\})=S h_{1}^{(3,1)}\left(\{1,2\}, v / \mathcal{C}^{2}\right)=\frac{3}{4}$.

\footnotetext{
${ }^{1} \mathrm{~A}$ value $f$ is additive if $f(v+w)=f(v)+f(w)$ for any two games $v, w$, where $v+w$ is defined as $(v+w)(S)=v(S)+w(S)$ for all $S$.

${ }^{2}$ Notice that player 2 in the game $\left(\{1,2\}, v / \mathcal{C}^{2}\right)$ represents coalition $C_{2}^{2}=\{4\}$.
} 
Stage 2: In this case, we have three games $v^{1}$, one for each coalition in $\mathcal{C}^{1}$. The game $\left(\{1\}, v^{1}\right)$ is defined as $v^{1}(\{1\})=S h_{1}^{(1,2)}\left(\{1,2\}, v^{2} / \mathcal{C}_{\{1,2,3\}}^{1}\right)=\frac{1}{4}$; whereas the game $\left(\{4\}, v^{1}\right)$ is defined as $v^{1}(\{4\})=S h_{3}^{(1)}\left(\{3\}, v^{2} / \mathcal{C}_{\{4\}}^{1}\right)=\frac{1}{4}$; and the game $\left(\{2,3\}, v^{1}\right)$ is defined as $v^{1}(\{2\})=S h_{2}^{(1,1)}\left(\{1,2\}, v^{2} / \mathcal{C}_{\{1,2\}}^{1}\right)=$ $\frac{1}{3}, v^{1}(\{3\})=S h_{2}^{(1,1)}\left(\{1,2\}, v^{2} / \mathcal{C}_{\{1,3\}}^{1}\right)=0$, and $v^{1}(\{2,3\})=S h_{2}^{(1,2)}\left(\{1,2\}, v^{2} / \mathcal{C}_{\{1,2,3\}}^{1}\right)=$ $\frac{1}{2}$.

Stage 3: In this case, we have $\phi_{1}(v)=S h_{1}^{(1)}\left(\{1\}, v^{1} / \mathcal{C}_{\{1\}}^{0}\right)=\frac{1}{4}, \phi_{2}(v)=$ $S h_{2}^{(1,1)}\left(\{2,3\}, v^{1} / \mathcal{C}_{\{2,3\}}^{1}\right)=\frac{5}{12}, \phi_{3}(v)=S h_{3}^{(1,1)}\left(\{2,3\}, v^{1} / \mathcal{C}_{\{2,3\}}^{1}\right)=\frac{1}{12}$, and $\phi_{4}(v)=S h_{4}^{(1)}\left(\{4\}, v^{1} / \mathcal{C}_{\{4\}}^{1}\right)=\frac{1}{4}$.

Hence, the final assignment of the value is $\phi(v)=\left(\frac{1}{4}, \frac{5}{12}, \frac{1}{12}, \frac{1}{4}\right)$. As opposed, the LSV assigns a value $\left(\frac{1}{4}, \frac{1}{4}, 0, \frac{1}{2}\right)$.

Notice that in the previous example player 3 is a null player ${ }^{3}$, and the value $\phi$ assigns him a positive payoff. Thus, it is deduced that the value $\phi$ does not satisfy the property of null player ${ }^{4}$. Notice, however, that we consider situations in which the players represent groups. The role of the null players could therefore, be important, since the power of negotiation among coalitions may depend on their size. Since, null players affect the size of the coalition, we should admit that they are not so null and thus, it is reasonable to assign them a positive payoff. For a detailed discussion see Vidal-Puga (2006) and Gómez-Rúa and Vidal-Puga (2007).

\section{Balanced per capita contributions}

Myerson (1980) defined the following properties:

Definition 2 A value $f$ satisfies Balanced Individual Contributions ${ }^{5}$ (BIC) if and only if

$$
f_{i}(N, v)-f_{i}(N \backslash\{j\}, v)=f_{j}(N, v)-f_{j}(N \backslash\{i\}, v)
$$

for all $i, j \in N$ and all TU game $(N, v)$.

\footnotetext{
${ }^{3} \mathrm{~A}$ null player is a player $i$ with $v(S \cup\{i\})=v(S)$ for all $S$.

${ }^{4} \mathrm{~A}$ value $f$ satisfies the property of null player if for any null player $i, f_{i}(N, v, \mathfrak{C})=0$.

${ }^{5}$ Myerson called it Balanced Contributions.
} 
Definition 3 Let $(N, v)$ be a TU game. Let $\alpha \in \mathbb{R}_{++}^{N}$. A value $f$ satisfies $\alpha$-Balanced Individual Contributions ${ }^{6}(\alpha-B I C)$ if and only if

$$
\frac{f_{i}(N, v)-f_{i}(N \backslash\{j\}, v)}{\alpha_{i}}=\frac{f_{j}(N, v)-f_{j}(N \backslash\{i\}, v)}{\alpha_{j}}
$$

for all $i, j \in N$.

Myerson (1980, Lemma 6) proved that, given $\alpha \in \mathbb{R}_{++}^{N}$, there exists a unique efficient value satisfying $\alpha$-balanced individual contributions.

Hart and Mas-Colell (1989) showed that this family of values coincides with the family of weighted Shapley values. See also Sánchez (1997) and Calvo and Santos (2000).

Proposition 4 (Hart and Mas-Colell, 1989, page 604): For any $\alpha \in \mathbb{R}_{++}^{N}$, $S h^{\alpha}$ is the only efficient value that satisfies $\alpha-B I C$.

Calvo et al. (1996) extended BIC to the context of games with a level structure as follows:

Definition 5 A value $f$ satisfies Balanced Group Contributions ${ }^{7}$ (BGC) if for all $C_{q}^{l}, C_{r}^{l} \in \mathcal{C}^{l}$ such that $C_{q}^{l}, C_{r}^{l} \subset C_{k}^{l+1} \in \mathcal{C}^{l+1}(l=0,1, \ldots, h-1)$, we have

$$
f_{C_{q}^{l}}(N, v, \mathfrak{C})-f_{C_{q}^{l}}\left(N \backslash C_{r}^{l}, v, \mathfrak{C}\right)=f_{C_{r}^{l}}(N, v, \mathfrak{C})-f_{C_{r}^{l}}\left(N \backslash C_{q}^{l}, v, \mathfrak{C}\right)
$$

for all $(N, v, \mathfrak{C}) \in L T U$.

This property states that for any two coalitions, $C_{q}^{l}$ and $C_{r}^{l}$ that belong to the same coalitions at higher levels, the contributions of $C_{q}^{l}$ to the total payoff of the members in $C_{r}^{l}$ must be equal to the contribution of $C_{r}^{l}$ to the total payoff of the members in $C_{q}^{l}$.

Calvo et al. (1996) characterized the level structure value with the property of $B G C$.

The formulation of $\alpha-B I C$ for the context of the TU games with a level structure, with $\alpha_{q}=\left|C_{q}^{l}\right|$ for all $C_{q}^{l} \in \mathcal{C}^{l}$ and all $l=0,1, \ldots, h-1$, is the following:

\footnotetext{
${ }^{6}$ Myerson used the equivalent notation $\lambda$-Balanced Contributions with $\lambda=\frac{1}{\alpha}$, where $\left(\frac{1}{\alpha}\right)_{i}=\frac{1}{\alpha_{i}}$.

${ }^{7}$ Calvo et al. called it Balanced Contributions.
} 
Definition 6 A value $f$ satisfies Balanced Per Capita Contributions (BPCC) if for all $C_{q}^{l}, C_{r}^{l} \in \mathcal{C}^{l}$ such that $C_{q}^{l}, C_{r}^{l} \subset C_{k}^{l+1} \in \mathcal{C}^{l+1}(l=0,1, \ldots, h-1)$, we have

$$
\frac{f_{C_{q}^{l}}(N, v, \mathfrak{C})-f_{C_{q}^{l}}\left(N \backslash C_{r}^{l}, v, \mathfrak{C}\right)}{\left|C_{q}^{l}\right|}=\frac{f_{C_{r}^{l}}(N, v, \mathfrak{C})-f_{C_{r}^{l}}\left(N \backslash C_{q}^{l}, v, \mathfrak{C}\right)}{\left|C_{r}^{l}\right|}
$$

for all $(N, v, \mathfrak{C}) \in L T U$.

This property ensures that for any two coalitions $C_{q}^{l}$ and $C_{r}^{l}$ that belong to the same coalitions at higher levels, the change per capita in the payoffs of the players in $C_{q}^{l}$ if $C_{r}^{l}$ leaves the game should be equal to the change per capita in the payoffs of the players in $C_{r}^{l}$ if $C_{q}^{l}$ leaves the game.

Proposition $7 \phi$ is efficient and satisfies BPCC.

Proof. It is straightforward to check that $\phi$ is efficient.

Now we prove that $\phi$ satisfies BPCC: Fix $(N, v, \mathfrak{C})$ with $\mathfrak{C}=\left\{\mathcal{C}^{0}, \ldots, \mathcal{C}^{h}\right\}$.

Given $C_{k}^{l+1} \in \mathcal{C}^{l+1}$, let $C_{q}^{l}, C_{r}^{l} \in \mathcal{C}^{l}$ such that $C_{q}^{l}, C_{r}^{l} \subset C_{k}^{l+1}$.

By definition of $\phi$, we have that

$\phi_{C_{q}^{l}}(N, v)-\phi_{C_{q}^{l}}\left(N \backslash C_{r}^{l}, v\right)=S h_{q}^{\delta\left(C_{q}^{l}\right)}\left(N_{k}^{l}, v^{l+1} / \mathcal{C}_{C_{k}^{l+1}}^{l}\right)-S h_{q}^{\delta\left(C_{q}^{l}\right)}\left(N_{k}^{l} \backslash\{r\}, v^{l+1} / \mathcal{C}_{C_{k}^{l+1} \backslash C_{r}^{l}}^{l}\right)$.

Notice that by definition of $\phi, v^{l+1}(T)$ does not depend on the presence of coalition $C_{r}^{l}$ in the player set when $T \subset C_{k}^{l+1} \backslash C_{r}^{l}$, so expression above can be restated as:

$\phi_{C_{q}^{l}}(N, v)-\phi_{C_{q}^{l}}\left(N \backslash C_{r}^{l}, v\right)=S h_{q}^{\delta\left(C_{q}^{l}\right)}\left(N_{k}^{l}, v^{l+1} / \mathcal{C}_{C_{k}^{l+1}}^{l}\right)-S h_{q}^{\delta\left(C_{q}^{l}\right)}\left(N_{k}^{l}, v^{l+1} / \mathcal{C}_{C_{k}^{l+1} \backslash C_{r}^{l}}^{l}\right)$.

Reasoning in a similar way, we obtain an analogous expression for $C_{r}^{l}$.

Since $S h^{\delta\left(C_{q}^{l}\right)}$ satisfies $\delta\left(C_{q}^{l}\right)$-BIC and $S h^{\delta\left(C_{r}^{l}\right)}$ satisfies $\delta\left(C_{r}^{l}\right)$-BIC (Proposition 4),

$$
\begin{aligned}
& \frac{S h_{q}^{\delta\left(C_{q}^{l}\right)}\left(N_{k}^{l}, v^{l+1} / \mathcal{C}_{C_{k}^{l+1}}^{l}\right)-S h_{q}^{\delta\left(C_{q}^{l}\right)}\left(N_{k}^{l}, v^{l+1} / \mathcal{C}_{C_{k}^{l+1} \backslash C_{r}^{l}}^{l}\right)}{\delta_{q}\left(C_{q}^{l}\right)} \\
= & \frac{S h_{r}^{\delta\left(C_{r}^{l}\right)}\left(N_{k}^{l}, v^{l+1} / \mathcal{C}_{C_{k}^{l+1}}^{l}\right)-S h_{r}^{\delta\left(C_{r}^{l}\right)}\left(N_{k}^{l}, v^{l+1} / \mathcal{C}_{C_{k}^{l+1} \backslash C_{q}^{l}}^{l}\right)}{\delta_{r}\left(C_{r}^{l}\right)} .
\end{aligned}
$$


Moreover, by definition of $\delta, \delta_{q}\left(C_{q}^{l}\right)=\left|C_{q}^{l}\right|$ and $\delta_{r}\left(C_{r}^{l}\right)=\left|C_{r}^{l}\right|$, thus we obtain the result.

\section{Characterization}

In this section we provide a characterization of the value $\phi$ using the property of Balanced Per Capita Contributions introduced in the previous section.

Theorem 8 An efficient value $f$ over the set of games with a level structure satisfies $B P C C$ if and only if $f=\phi$.

Proof. We know by Proposition 7 that $\phi$ is efficient and satisfies BPCC. Assume there exist two efficient values $f^{1}$ and $f^{2}$ satisfying BPCC. Fix $h \geq 1$. We will prove that $f_{C_{q}^{l}}^{1}(v)=f_{C_{q}^{l}}^{2}(v)$ for all $v \in L T U, l \in\{0, \ldots, h\}$ and $C_{q}^{l} \in \mathcal{C}^{l}$. This is enough to prove the result because the $C_{q}^{l}$ are singletons for $l=0$.

We will prove the result by induction on the level. Consider level $h$, $\mathcal{C}^{h}=\{N\}$. Since $f^{1}$ and $f^{2}$ are efficient, we have that $f_{N}^{1}(v)=f_{N}^{2}(v)=v(N)$ for all $v$.

Let $l \in\{1, \ldots, h\}$ and assume that the result holds for level $k, k \geq l$, i.e.

$$
f_{C_{q}^{k}}^{1}(v)=f_{C_{q}^{k}}^{2}(v)
$$

for all $C_{q}^{k} \in \mathcal{C}^{k}$ with $l \leq k \leq h$.

Let $C_{q}^{l} \in \mathcal{C}^{l}$. Denote $\mathcal{Q}^{l-1}=\left\{C_{r}^{l-1} \in \mathcal{C}^{l-1}: C_{r}^{l-1} \subset C_{q}^{l}\right\}$.

We use an induction argument on the cardinal of $\mathcal{Q}^{l-1}$.

Assume that $\left|\mathcal{Q}^{l-1}\right|=1$, say $\mathcal{Q}^{l-1}=\left\{C_{r}^{l-1}\right\}$. Hence, $C_{r}^{l-1}=C_{q}^{l}$ and by induction hypothesis:

$$
f_{C_{r}^{l-1}}^{1}(v)=f_{C_{q}^{l}}^{1}(v)=f_{C_{q}^{l}}^{2}(v)=f_{C_{r}^{l-1}}^{2}(v) .
$$

Assume that the result holds for $\left|\mathcal{Q}^{l-1}\right|=m-1$. Now we prove that it holds for $\left|\mathcal{Q}^{l-1}\right|=m$.

Suppose that $\left|\mathcal{Q}^{l-1}\right|=m \geq 2$, say $\mathcal{Q}^{l-1}=\left\{C_{1}^{l-1}, \ldots, C_{m}^{l-1}\right\}$. Let $M=$ $\{1, \ldots, m\}$.

Let $C_{r}^{l-1}, C_{s}^{l-1} \in \mathcal{Q}^{l-1}$. Under BPCC,

$$
\frac{f_{C_{r}^{l-1}}^{1}(N, v)-f_{C_{r}^{l-1}}^{1}\left(N \backslash C_{s}^{l-1}, v\right)}{\left|C_{r}^{l-1}\right|}=\frac{f_{C_{s}^{l-1}}^{1}(N, v)-f_{C_{s}^{l-1}}^{1}\left(N \backslash C_{r}^{l-1}, v\right)}{\left|C_{s}^{l-1}\right|}
$$


and

$$
\frac{f_{C_{r}^{l-1}}^{2}(N, v)-f_{C_{r}^{l-1}}^{2}\left(N \backslash C_{s}^{l-1}, v\right)}{\left|C_{r}^{l-1}\right|}=\frac{f_{C_{s}^{l-1}}^{2}(N, v)-f_{C_{s}^{l-1}}^{2}\left(N \backslash C_{r}^{l-1}, v\right)}{\left|C_{s}^{l-1}\right|} .
$$

Moreover, by induction hypothesis on $\left|\mathcal{Q}^{l-1}\right|$ we have that $f_{C_{r}^{l-1}}^{1}\left(N \backslash C_{s}^{l-1}, v\right)=$ $f_{C_{r}^{l-1}}^{2}\left(N \backslash C_{s}^{l-1}, v\right)$ and $f_{C_{s}^{l-1}}^{1}\left(N \backslash C_{r}^{l-1}, v\right)=f_{C_{s}^{l-1}}^{2}\left(N \backslash C_{r}^{l-1}, v\right)$.

Taking into account these expressions and operating with (1) and (2), we have that

$$
\frac{f_{C_{r}^{l-1}}^{1}(N, v)-f_{C_{r}^{l-1}}^{2}(N, v)}{\left|C_{r}^{l-1}\right|}=\frac{f_{C_{s}^{l-1}}^{1}(N, v)-f_{C_{s}^{l-1}}^{2}(N, v)}{\left|C_{s}^{l-1}\right|}
$$

and so,

$$
f_{C_{r}^{l-1}}^{1}(N, v)-f_{C_{r}^{l-1}}^{2}(N, v)=\frac{\left|C_{r}^{l-1}\right|}{\left|C_{s}^{l-1}\right|}\left[f_{C_{s}^{l-1}}^{1}(N, v)-f_{C_{s}^{l-1}}^{2}(N, v)\right]
$$

for all $C_{r}^{l-1}, C_{s}^{l-1} \in \mathcal{Q}^{l-1}$.

Applying the induction hypothesis on levels, we have that $f_{C_{q}^{l}}^{1}(N, v)=$ $f_{C_{q}^{l}}^{2}(N, v)$. Fix $C_{s}^{l-1} \in \mathcal{Q}^{l-1}$. Therefore,

$$
\begin{aligned}
0= & f_{C_{q}^{l}}^{1}(N, v)-f_{C_{q}^{l}}^{2}(N, v)=\sum_{C_{r}^{l-1} \in \mathcal{Q}^{l-1}}\left[f_{C_{r}^{l-1}}^{1}(N, v)-f_{C_{r}^{l-1}}^{2}(N, v)\right] \\
= & \text { (3) } \sum_{C_{r}^{l-1} \in \mathcal{Q}^{l-1}}\left[\frac{\left|C_{r}^{l-1}\right|}{\left|C_{s}^{l-1}\right|}\left(f_{C_{s}^{l-1}}^{1}(N, v)-f_{C_{s}^{l-1}}^{2}(N, v)\right)\right] \\
= & {\left[f_{C_{s}^{l-1}}^{1}(N, v)-f_{C_{s}^{l-1}}^{2}(N, v)\right] \sum_{C_{r}^{l-1} \in \mathcal{Q}^{l-1}} \frac{\left|C_{r}^{l-1}\right|}{\left|C_{s}^{l-1}\right|} . }
\end{aligned}
$$

and since $\sum_{C_{r}^{l-1} \in \mathcal{Q}^{l-1}} \frac{\left|C_{r}^{l-1}\right|}{\left|C_{s}^{l-1}\right|}>0$, we have that $f_{C_{s}^{l-1}}^{1}(N, v)=f_{C_{s}^{l-1}}^{2}(N, v)$. Replacing this expression in (3), we obtain that $f_{C_{r}^{l-1}}^{1}(N, v)=f_{C_{r}^{l-1}}^{2}(N, v)$ for all $C_{r}^{l-1} \in \mathcal{Q}^{l-1}$.

Hence, we have proved that $f_{C_{r}^{l-1}}^{1}(N, v)=f_{C_{r}^{l-1}}^{2}(N, v)$ for any $C_{r}^{l-1} \in$ $\mathcal{C}^{l-1}$. By applying the induction on $l$, we have $f_{C_{r}^{l}}^{1}(N, v)=f_{C_{r}^{l}}^{2}(N, v)$ for all $C_{r}^{l} \in \mathcal{C}^{l}$ and all $l \geq 0$. In particular, for $l=0$, we have $f_{C_{r}^{0}}^{1}(N, v)=f_{C_{r}^{0}}^{2}(N, v)$ for all $C_{r}^{0} \in \mathcal{C}^{0}$. Since $C_{q}^{0}=\{q\}$ for all $C_{q}^{0} \in \mathcal{C}^{0}$, we have the result. 


\section{References}

[1] Aumann, R. and Drèze, J. (1974) Cooperative games with coalition structure. International Journal of Game Theory 3, 217-237.

[2] Calvo, E., Lasaga, J. and Winter, E. (1996) The principle of balanced contributions and hierarchies of cooperation. Mathematical Social Sciences $31,171-182$.

[3] Calvo, E. and Santos, J.C. (2000) Weighted weak semivalues. International Journal of Game Theory 29, 1-9.

[4] Gómez-Rúa, M. and Vidal-Puga, J. J. (2008) The axiomatic approach to three values in games with coalition structure. MPRA paper number 8904. Available at http://econpapers.repec.org/paper/pramprapa/8904.htm

[5] Hamiache G. (2006) A value for games with coalition structures. Social Choice and Welfare 26, 93-105.

[6] Hart, S. and Kurz, M. (1983) Endogenous formation of coalitions. Econometrica 51 (4), 10471064.

[7] Hart, S. and Mas-Colell, A. (1989) Potencial, value and consistency. Econometrica 57 (3), 589-614.

[8] Herings, P.J.J., van der Laan, G. and Talman, D. (2008) The average tree solution for cycle-free graph games. Games and Economic Behaviour $62,77-92$.

[9] Kalai, E., and Samet, D. (1987) On weighted Shapley values. International Journal of Game Theory 16, 205-222.

[10] Kalai, E., and Samet, D. (1988) Weighted Shapley values. In: A. Roth, ed., The Shapley value: Essays in honor of L. Shapley (Cambrige), Cambrige University Press, 83-99.

[11] Kamijo Y. (2007) A collective value: a new interpretation of a value and a coalition structure. Waseda University. 21 COE-GLOPE Working Paper Series 27. 
[12] Levy, A. and McLean, R.P. (1989) Weighted coalition structure values. Games and Economic Behavior 1, 234-249.

[13] Myerson, R.B. (1977) Graphs and cooperation in games. Mathematics of Operations Research 2, 225-229.

[14] Myerson, R.B. (1980) Conference structures and fair allocation rules. International Journal of Game Theory 9, 169-182.

[15] Owen, G. (1977) Values of games with a priori unions. In: Henn, R., Moeschlin, O. (eds.) Essays in mathematical economics and game theory (Berlin) Heidelberg NewYork: Springer, 76-88.

[16] Pérez-Castrillo, D. and Wettstein, D. (2001) Bidding for the surplus: A non-cooperative approach to the Shapley value. Journal of Economic Theory 100, 274-294.

[17] Sánchez, F. (1997) Balanced contributions axiom in the solution of cooperative games. Games and Economic Behavior 20, 161-168.

[18] Shapley, L. S. (1953a) Additive and Non-Additive Set Functions. Ph.D. Dissertation, Princeton University.

[19] Shapley, L. S. (1953b) A value for n-person games. In: Huhn, H.W.,Tucker, A.W. (eds.) Contributions to the theory of games II. Annals of Mathematics Studies 28, pp. 307-317. Princeton: Princeton University Press.

[20] Vidal-Puga, J. (2005) Implementation of the levels structure value. Annals of Operations Research 137(1), 191-209.

[21] Vidal-Puga, J. (2006) The Harsanyi paradox and the "right to talk" in bargaining among coalitions. EconWPA Working paper number 0501005. Available at http://ideas.repec.org/p/wpa/wuwpga/0501005.html.

[22] Winter, E. (1989) A value for cooperative games with level structure of cooperation. International Journal of Game Theory 18, 227-240. 